\title{
MULTI-FREQUENCY VLBI OBSERVATIONS OF 3C390.3
}

\author{
E. PREUSS ${ }^{1}$, W. ALEF ${ }^{1}$, K. I. KELLERMANN ${ }^{2}$ \\ ${ }^{1}$ Max-Planck-Institut für Radioastronomie, Auf dem Hügel 69, \\ 53121 Bonn, Germany \\ 2 National Radio Astronomy Observatory, 520 Edgemont Road, \\ Charlottesville, VA 22903, USA
}

\begin{abstract}
We have mapped the broad line radio galaxy 3 C390.3 at 1.3, 6 , and $18 \mathrm{~cm}$ wavelengths with resolutions ranging from 0.2 to 2.7 mas. Our new $6 \mathrm{~cm}$ image is consistent with a stationary bright region located 4 to 5 mas from the core and motion of the other features with apparent velocities of 0.4 to $0.7 \mathrm{mas} / \mathrm{yr}$. Our high resolution $1.3 \mathrm{~cm}$ image indicates that the 'jet' breaks up into 5 or more distinct features.
\end{abstract}

The $\mathrm{N}$ galaxy $3 \mathrm{C} 390.3(1845+797)$ is one of the closest radio galaxies with observed component motion and is also one of the nearest objects of FR type II $\left(\mathrm{z}=0.057 ; 1 \mathrm{mas}=1.5 \mathrm{pc} ; \mathrm{H}_{0}=50 \mathrm{~km} / \mathrm{s} / \mathrm{Mpc}, \mathrm{q}_{0}=0.5\right)$. VLA observations with arcsec resolution show two lobes, each with compact structure, and a weak one-sided thin jet joining the NW lobe to a central core (Alef et al. 1995, Leahy and Perley 1995).

Our previous $6 \mathrm{~cm}$ VLBI observations made between 1978 and 1989 suggested the ejection of multiple radio components at a rate of about once every four years with an apparent component motion of 0.7 mas/year $(v / c=3.5)$, but we also found evidence for a stationary region of enhanced emission located between 4 and 5 mas from the 'core' (Alef et al. 1995, Alef et al. 1988).

In order to clarify the morphology and apparent kinematics of the central region of $3 \mathrm{C} 390.3$ we have made new observations at $1.3,6$, and 18 $\mathrm{cm}$ which we report here.

The $6 \mathrm{~cm}$ observations, made with the VLBA in 1995.29, extends the range of the previous sequence of observations by an additional 6 years. The map (Fig. 1) shows the same general features seen previously. As before we 


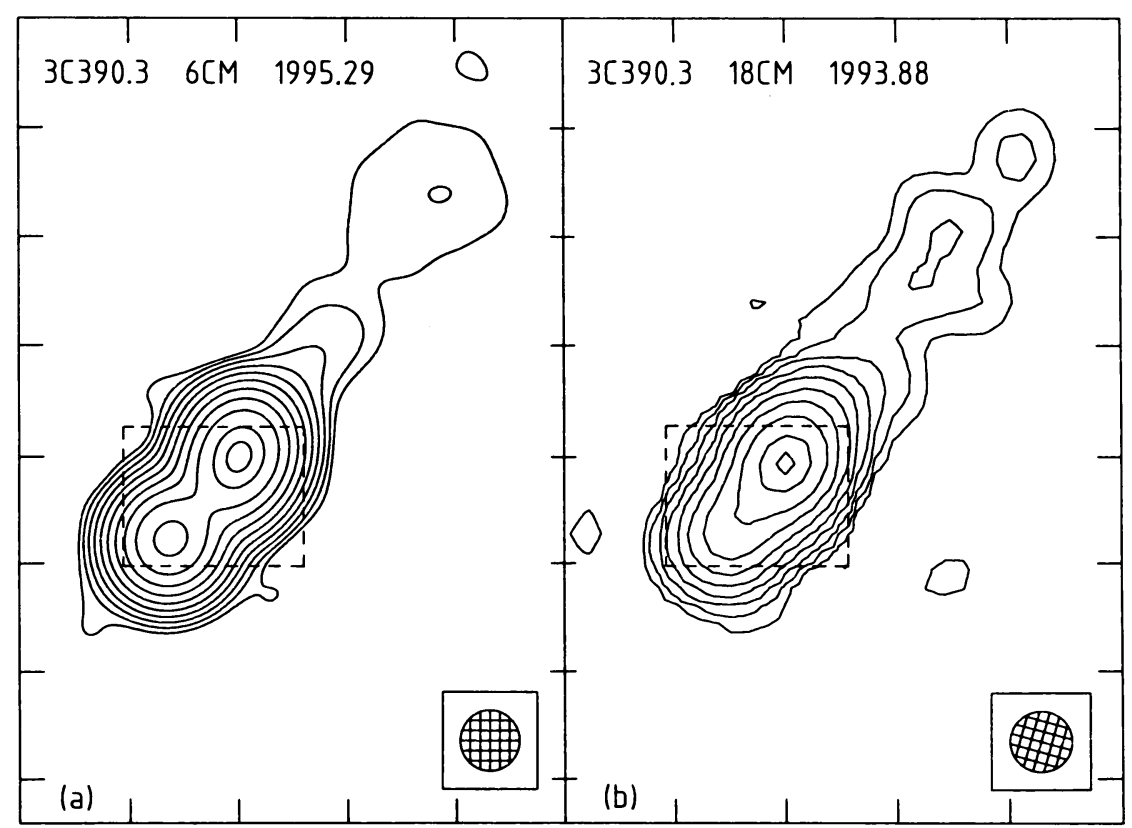

Figure 1. VLBI maps of $3 \mathrm{C} 390.3$ at a resolution of 2.7 mas. a) $\lambda=6 \mathrm{~cm}$ : contour intervals are at $0.125,0.25,0.5,1,2,4,8,16,32,64$, and $90 \%$ of the peak brightness of $350 \mathrm{mJy} /$ beam. b) $\lambda=18 \mathrm{~cm}$ : Contour intervals are the same as in (a) except the lowest contour level is at $0.25 \%$ of the peak brightness of $268 \mathrm{mJy} /$ beam. The spacing between tick marks is 5 mas. The dashed rectangles mark the area covered by Fig. 2

see that the brightest jet feature is located $\sim 5.3$ mas away from the core, but it has now become as strong as the core component, whereas in 1989 and earlier it was much weaker.

Alef et al. (1995) noted that in the 1989 image, the jet component at 4-5 mas from the core appears to bend through an angle of $\sim 3^{\circ}$. The new data show a sharper bend of $\sim 12^{\circ}$ which obviously mirrors the even sharper bend in the ridge line of the $1.3 \mathrm{~cm}$ image (Fig. 2). The faint features seen at about 12 and 20 mas from the core may be identified with features seen in the earlier observations if they have moved at a rate of 0.5 to $0.6 \mathrm{mas} / \mathrm{yr}$; but the association with the features present six or more years ago is not unambiguous.

The $18 \mathrm{~cm}$ image, shown in Fig.1b, was obtained from a 15 station observation using the VLBA plus antennas at Green Bank (140 ft), Jodrell Bank (Mk I), Effelsberg, Medicina and Onsala, and was intended to trace intermediate scale structure whose surface brightness is too low to be seen in the $6 \mathrm{~cm}$ images. The $18 \mathrm{~cm}$ map traces the jet feature out to a distance of 20 mas $(30 \mathrm{pc})$ from the core. Comparison with the $6 \mathrm{~cm}$ image with the same resolution shows the outer jet to be much brighter at the longer 


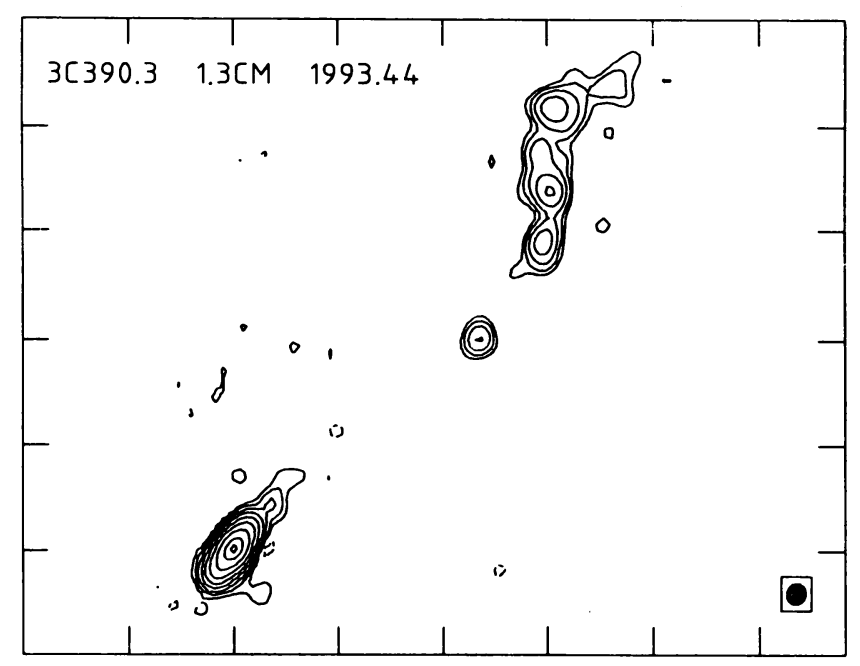

Figure 2. VLBI map of $3 C 390.3$ at $\lambda=1.3 \mathrm{~cm}$ with a resolution of 0.2 mas. Contour levels are at $-0.5,0.5,1,2,4,8,16,32,64$, and $90 \%$ of the peak brightness of $143 \mathrm{mJy} /$ beam. The spacing between tick marks is 1 mas.

wavelength.

The $1.3 \mathrm{~cm}$ observations were made in 1993.44 and used all antennas of the VLBA (except Mauna Kea, Owens Valley and Saint Croix) plus the Effelsberg, Medicina, Noto and Onsala $(20 \mathrm{~m})$ antennas. This global array gives a resolution of $\sim 0.2$ mas so that features as small as $0.3 \mathrm{pc}$ are well resolved. The $1.3 \mathrm{~cm}$ image (Fig. 2) has a very different appearance from the lower resolution $6 \mathrm{~cm}$ image. At $1.3 \mathrm{~cm}$, the strong $\mathrm{SE}$ component, which we identify with the core, is resolved. The inner part of the jet is clearly visible pointing toward a jet feature 2.5 mas away. Apparently the jet is formed on a scale $\leq 1 \mathrm{pc}$, and remains remarkably well collimated over more than $100 \mathrm{kpc}$. The NW jet feature, which we identify with the bright $6 \mathrm{~cm}$ feature located at the same position, shows remarkable detail and appears to break up into at least five discrete components which lie along a curved locus. At $\sim 5$ mas from the core, the jet shows a sharp bend and then points toward the extended jet $\left(\mathrm{PA} \sim-35^{\circ}\right)$.

The NRAO is a facility of the National Science Foundation and is operated by Associated Universities Inc. under a cooperative agreement.

\section{References}

Alef, W., Götz, M.M.A., Preuss, E., Kellermann, K.I. (1988) Structural changes in the nucleus of the double radio galaxy 3C390.3, Astron. Astrophysics, 182, pp. 53-56

Alef, W., Wu, S.Y., Preuss, E., Kellermann, K.I. and Qiu, Y.H. (1995) 3C390.3: a Lobe-Dominated Radio Galaxy with a Possible Superluminal Nucleus, Astron. Astrophysics, in press

Leahy, J.P. and Perley, R.A. (1995) The Jets and Hotspots of 3C390.3, MNRAS , in Press 